\title{
INICIATIVA DE SALUD GLOBAL DEL WABASH COLLEGE: EXPERIENCIA DE INMERSIÓN EN EL PERÚ
}

\author{
WABASH COLLEGE GLOBAL HEALTH INITIATIVE: \\ IMMERSION EXPERIENCE IN PERU \\ Jorge Cárdenas-Callirgos ${ }^{1,2}$, Eric J. Wetzel'
}

\begin{abstract}
RESUMEN
La misión de la Iniciativa de Salud Global del Wabash College (WC) es transformar la vida de los estudiantes y voluntarios a través de la educación, la investigación y el servicio en salud pública, buscando generar un impacto positivo en las comunidades desatendidas a nivel global. El programa, financiado gracias a colaboradores filántropos, provee a los estudiantes y voluntarios provenientes de diversas especialidades una experiencia local e internacional de construcción de la comunidad que los preparan para ser líderes globales. En el 2,016 después de varios años de actividades con estudiantes de la Facultad de Medicina Humana de la Universidad Ricardo Palma, se firma un acuerdo entreWC y la URP. Los estudiantes tienen la oportunidad de participar en una amplia gama de actividades cocurriculares, incluyendo: inmersión en salud global; pasantías internacionales, regionales y locales; así como creación de redes y asesoramiento académico bajo tutoría de catedráticos, compañeros y profesionales de este campo.
\end{abstract}

Palabras clave: Salud global; Enfermedades Parasitarias desatendidas; Perú. (fuente: DeCS BIREME)

\begin{abstract}
The mission of the Wabash College Global Health Initiative is to transform the lives of students and volunteers through public health education, investigation and service, and in so doing to effect positive change in underserved communities globally. The program, funded through philanthropic partners, provides students and volunteers from all majors with international and local community-building experiences that prepare them to be global leaders. Students and volunteers have the opportunity to participate in a wide range of co-curricular activities including: global health immersion; international, regional, and local internships; and networking and mentoring from faculty, peers, and professionals in the field.
\end{abstract}

Key words: Global health; Neglected parasitic diseases; Peru. (source: MeSH NLM)

\section{INTRODUCCIÓN}

En la región neotropical enfrentamos nuevos desafíos de cara a conseguir que el sistema de salud sea accesible a las poblaciones más vulnerables, en este contexto surge la importancia de desarrollar un programa de salud global que abarcando no solo la salud pública, sino también la animal y ambiental bajo el enfoque integrador de Una sola Salud (One Health) asuma la misión de agrupar esfuerzos multidisciplinarios en miras a desarrollar propuestas multisectoriales, que contribuyan a edificar una cultura solidaria donde todos tengan acceso a condiciones sanitarias dignas y adecuadas a sus necesidades inmediatas.
La salud global podría definirse como un área de estudio que se enfoca en los problemas y determinantes de la salud que trascienden las fronteras de las naciones y demandan acciones de cooperación y que bajo una perspectiva global aborda la salud pública promoviendo la cooperación interdisciplinaria en busca de soluciones a varios niveles contribuyendo a una cultura de prevención sanitaria'.

La Iniciativa de Salud Global (Global Health Initiative - GHI) del Wabash College comenzó a desplegarse en tierras peruanas a principios del 2009 mediante la elaboración de una red de cooperadores nacionales e internacionales quienes unieron esfuerzos para

${ }^{1}$ Global Health Initiative, Wabash College, Indiana, USA.

2Asociación Peruana de Helmintología e Invertebrados Afines (APHIA), Lima, Perú.

Correspondencia: Jorge Cárdenas Callirgos. Dirección: In-country Coordinator, Perú. Global Health Initiative. Wabash College, Indiana, USA. Correo: jmcardenasc.ghi@gmail.com 
realizar un esfuerzo solidario en miras a cubrir las necesidades básicas de salubridad en una de las zonas más afectadas de la ciudad de Lima que han quedado atrapadas en el ciclo de la pobreza, siendo la más extendida a lo largo del territorio nacional liderada por una institución extranjera, a diferencia del programa liderado por la Universidad Peruana Cayetano Heredia $(\mathrm{UPCH})^{2}$ que si bien se encuentra focalizado en lo referido a sus zonas de intervención, la UPCH ha sido pionera en desarrollar un sistema de capacitación académica de excelente nivel encarnado en la realidad nacional ${ }^{3}$. De esta manera las intervenciones mediante talleres y campañas se concretizaron a inicios del 2010 en la zona conocida como Nueva Rinconada (Pamplona Alta, San Juan de Miraflores) y a partir de esa experiencia se fue extendiendo a diferentes comunidades de los departamentos del Perú, siendo los primeras Ancash, Huánuco, San Martin y Loreto. Esto se puede atribuir a la extensa red de instituciones, colaboradores y voluntarios institucionales que hemos establecido en los últimos años 4 .

\section{EDUCACIÓN}

El objetivo académico de GHI - Perú es educar a nuestro equipo de trabajo sobre diversos problemas de salud global desde un enfoque multidisciplinario. Nuestros voluntarios y colaboradores viven una experiencia de inmersión donde no sólo aprenden, sino que también sirven (y al hacerlo, aprenden de una manera más plena y diferente). Los problemas de salud en los que se ha focalizado $\mathrm{GHI}$ desde sus inicios son aquellos que trascienden las fronteras nacionales, afectan desproporcionadamente a la población desatendida y necesariamente precisan ser abordadas desde una perspectiva multidisciplinaria.

\section{INVESTIGACIÓN}

Los principales objetivos del área de investigación de GHI - Perú son entender los determinantes de la salud tanto sociales como biológicos en comunidades desatendidas desde un triple enfoque que abarca las áreas de Salud Global Publica, Salud Global Animal y Salud Global Ambiental \& Ecotoxicología, elaborando en su conjunto una perspectiva holística que seenmarca en el concepto One Health ${ }^{5}$. De manera concreta estamos enfocados en temas prioritarios como: agua, saneamiento, medio ambiente, zoonosis parasitarias (transmitidas tanto por animales domésticos como silvestres así como peces) y más recientemente enfermedades crónicas no transmisibles, siendo estas nuestras principales líneas de acción debido a que afectan la salud materno infantil y repercuten en el estado de salud de toda la comunidad afectada.

Hay importantes puntos de interrelación entre los objetivos de esta área con los fines educativos de $\mathrm{GHI}$, ya que estamos comprometidos a trabajar en coordinación con nuestros colaboradores locales para promover espacios de formación y discusión en salud preventiva, facilitando información relevante basada en los resultados de nuestras intervenciones y desarrollando estrategias de educación sanitaria fomentando la participación protagónica de nuestros agentes comunitarios, buscando por medio de talleres y grupos de trabajo posibles soluciones conjuntas a los principales problemas de salud de la comunidad.

\section{SERVICIO}

La meta del área de servicio de GHI - Perú está íntimamente relacionada con los fundamentos de las áreas de Educación e Investigación. Parte de la misión de $\mathrm{GHI}$ es transformar las vidas de los voluntarios y colaboradores al estos involucrase en algunos aspectos de la temática en salud pública global en el Neotrópico. Por otra parte buscamos lograr un impacto positivo en la situación sanitaria de las comunidades desatendidas que son parte de nuestra área de acción.

En muchos sentidosestos postulados son consecuentes con la misión del Wabash College de educar a sus estudiantes a "actuar con responsabilidad" y "vivir humanamente". Por supuesto, no sólo la interacción con las características propias de la problemática sanitaria de estas comunidades son la principal fuente de formación de los participantes de nuestros proyectos sino particularmente la interacción con la persona humana, con el beneficiario de nuestras actividades de proyección, es la genera el mayor impacto en la formación humana y profesional de los miembros de nuestro equipo.

\section{POBREZA Y ENFERMEDADES INFECCIOSAS: EXPERIENCIA DE CAMPO}

Las limitaciones en infraestructura y servicios de salud vinculadas a la pobreza se tornan un caldo de cultivo para la aparición de diversas enfermedades infecciosas $^{6}$. Si bien nuestras experiencia en la actualidad se extiende por varios departamentos de nuestro país, las intervenciones en la zona de Pamplona Alta (SJM, Lima) han sido un espacio privilegiado para aplicar nuestros conocimientos sobre la dinámica de transmisión de las enfermedades infecciosas parasitarias en miras contribuir a levantar una line 
base de información sanitaria que incluye: factores de riesgo epidemiológico, estado nutricional y prevalencia de parasitosis (pediculosis, toxocariasis, oxyuriasis y parasitosis gastrointestinal), estas actividades han sido realizadas por diversos equipos de trabajo formados por voluntarios y siempre acompañados de talleres educativos participativos que procuran contribuir al desarrollo sostenible de la comunidad ${ }^{7-11}$.

\section{ENFERMEDADES NEOTROPICALES DESATENDIDAS: MISIÓN SOLIDARIA}

La experiencia de Lima se ha podido replicar en otras regiones focalizando nuestros esfuerzos de intervención e investigación en las llamadas NTD's (Neglected Tropical Diseases) conocidas como Enfermedades Tropicales Desatendidas, en su mayoría de carácter infeccioso (virales, bacterianas, micóticas y parasitarias, tanto asociadas a protozoarios y metazoarios (helmintos y artrópodos) que son marcadamente prevalentes en el Neotrópico gracias a las características ecológicas y a las condiciones socioculturales que caracterizan nuestra región. En este contexto ecoepidemiológico, el Perú se nos presenta como un mosaico de microclimas y patrones biogeográficos que enmarcados en la extendida situación de pobreza y marginalidad de nuestras comunidades fomentan la presencia de diversas enfermedades infecciosas desatendidas (EID) de entre las cuales las parasitarias ocupan un lugar preeminente (Enfermedad de Chagas, Leishmaniasis, Hidatidosis, Cisticercosis se encuentran entre las más conocidas) ${ }^{12}$, así como la emergencia de nuevos agentes etológicos parasitarios pobremente estudiados y en muchos casos completamente desconocidos a pesar de haber sido reportados desde décadas atrás ${ }^{13}$. En este esfuerzo hemos logrado realizar actividades en la región andina ${ }^{14-15}$ y la selva amazónica ${ }^{16}$ en miras a ser fieles a nuestra misión de buscar el desarrollo integral y sostenible de la comunidades vulnerables en las periferias de nuestro país ${ }^{17}$.
Esta experiencia nos ofrece la oportunidad que participantes provenientes de diversas disciplinas (no solamente del área de las ciencias de salud sino también de otras vertientes académicas) puedan enriquecer su capacidad de trabajo en equipo y análisis crítico al desarrollar y participar de proyectos en salud global que les permiten explorar múltiples perspectivas disponiéndolos a ser agentes de cambio participando activamente en el contexto cultural actual marcado por la presencia de comunidades globalizadas.

Por lo tanto nuestra misión se podría resumir en contribuir al desarrollo sostenible e integral de las comunidades vulnerables del Perú por medio de la investigación, la educación y el servicio, generando espacios de formación humana dirigidos a voluntarios, universitarios y profesionales, provenientes de diversos ámbitos académicos así como a los líderes y agentes del servicio de las comunidades periurbanas y rurales que atendemos dándole un rostro humano a la investigación científica y promoviendo el desarrollo integral de toda la persona humana.

La Iniciativa Global de Salud tiene como fin incentivar que nuestros voluntarios y colaboradores tengan la experiencia humana de vivir el servicio solidario en el contexto actual marcado por los retos que nos exige una cultura que se edifica de espaldas al otro y cultivar valores que generen nuevos líderes que hagan la diferencia en la vida de los todos aquellos que viven en condiciones de vida menos humanas para trabajar en miras a ofrecerles condiciones de vida más humanas contribuyendo así a la construcción de una cultura solidaria.

Financiamiento: Apoyo económico por el Wabash College, Indiana, US.

Conflicto de interés: Los autores declaran no tener conflicto de interés en la publicación de este artículo.

Recibido: 06 de enero del 2017

Aprobado: 23 de enero del 2017

\section{REFERENCIAS BIBLIOGRÁFICAS}

1. Skolnik R. Global Health 101. 2nd ed. Jones \& Bartlett Learning. United States of America; 2012.

2. Villafuerte-Galvez J, Curioso WH. Teaching global health at the frontlines: A multi-disciplinary course in Peru presents basic concepts to students. PLoS Med. 2007;4(6):e130.

3. Curioso WH, Lazo-Escalante M, Gotuzzo E, García HH, Gilman RH, García PJ. Entrenando a la nueva generación de estudiantes en salud global en una universidad peruana. Rev Peru Med Exp Salud Publica. 2008;25(3):269-273.
4. Liberal Arts Plus Global Health. Wabash College.[Internet]. 2017 [cited 2017 Apr 5]. Available from: https://www.wabash.edu/plus/health

5. One Health: Food and Agriculture of the United Nations Strategic. Action Plan [Brochure]. 2011. Rome.

6. Alsan MM, Westerhaus M, Herce M, Nakashima K, Farmer PE. Poverty, global health, and infectious disease: Lessons from Haiti and Rwanda. Infect Dis Clin North Am.. 2011;25(3):611-22.

7. Cárdenas-Callirgos J, Machuca - Bandach A, Jara - Richard M, Hidalgo 
- Portilla S, Meza - Zavala A, Hinostroza - Gormas S, Deharo E, Wetzel E. Pediculosis infantil en el AA.HH El Trébol - Pamplona Alta, Lima Perú y su relación con índices de malnutrición y factores de riesgo epidemiológico. En: Iannacone, José (Ed). II Congreso Internacional de Parasitología Neotropical; 2010 Noviembre 09 al 13; Universidad Ricardo Palma, Lima, Perú; 2010.

8. Cárdenas-Callirgos, J.; Rojas-Camacho, F.; Tineo-Valencia, M.; TorresMárquez, A.; Vilcapoma-Serpa, P.; Zarate-Vaella, T.; Puray-Chávez, N.; Wetzel, E. Presencia de agentes etiológicos de toxocariosis y evaluación de factores de riesgo epidemiológico en el AA.HH El Trébol - Pamplona Alta, Lima-Perú. En: lannacone, José (Ed). II Congreso Internacional de Parasitología Neotropical; 2010 Noviembre 09 al 13; Universidad Ricardo Palma, Lima, Perú; 2010.

9. Cárdenas-Callirgos, J., G. Gonzalez-Aspajo, F. Rojas-Camacho, M. TineoValencia, A. Torres-Márquez, P. Vilcapoma-Serpa, T. Zarate-Vaella, N. Puray-Chávez, E. Wetzel, E. Deharo. Correlation between toxocariasis in children and dogs at El Trebol, Pamplona Alta, San Juan de Miraflores a human settlement of Lima (Peru). En: Meeting of the French Society of $\exists$ Parasitology; February, 2012; Toulouse, France; 2012.

10. Quiñones-Laveriano, D. K. Patiño-Calla, S. Fustamante-Burga, P. Infante-Bustamante, C. Ruiz-del-Castillo, A. Palacios-Huan, F. VilcaSalguero, J. Quispe-Parcco, P. Luzón-Lopez, J. Salazar-Aguirre, S. CarrascoTejerina, C. Reinoso-Trabucco, E. Miraval-Cabrera, R. Valdivia-Cabrera, J. Sandoval-Pérez, A. Cvetković-Vega, G. Oliva-Segura, B.Romero-Aponte, J. Cárdenas-Callirgos, E. Wetzel. 2012. Intestinal parasite prevalence and hemoglobin levels of a marginal urban population as part of a health campaign in Pamplona Alta, San Juan de Miraflores 2012. En: lannacone, J. \& Cárdenas-Callirgos, J. (Eds). 2012. III Congreso Internacional de Parasitología Neotropical (III COPANEO); The Biologist (Lima), vol 10 (2), Suplemento Especial 2; 2012 Noviembre 5 al 10; Lima, Perú; 2012.

11. Patiño-Calla, K., P. Puescas-Sánchez, M. Cárdenas- Carranza, O. Cáceres-Torres, I. Cornejo-Bermudez, J. Cárdenas-Callirgos, C. LunaMuñoz, V. Durand-Tuanama \& E. Wetzel. Clinic-epidemiological characteristics and factors associated with Pediculosis capitis in a public school, Lima 2012. En: lannacone, J. \& Cárdenas-Callirgos, J. (Eds). 2012. III Congreso Internacional de Parasitología Neotropical (III COPANEO); The Biologist (Lima), vol 10 (2), Suplemento Especial 2; 2012 Noviembre 5 al 10; Lima, Perú; 2012.
12. Cabezas - Sanchez, C. Enfermedades infecciosas desatendidas: un permanente reto para la salud pública y la equidad en el Perú. Rev Peru Med Exp Salud Publica. 2014;31(2):326-335 .

13. Tantaleán M. Nuevos helmintos de importancia médica en el Perú. Rev. Per. Med. Trop. UNMSM. 1994;8:87-91.

14. Mayta - Calderon, M., J. Chambi - Torres, R. Uscata - Barrenechea, J. Huaccho - Rojas, J. Cárdenas - Callirgos, \& E. Wetzel. 2012. Parasitosis gastrointestinal en la comunidad rural de Llupa, Ancash-Peru, 2012. En: lannacone, J. \& Cárdenas-Callirgos, J. (Eds). 2012. III Congreso Internacional de Parasitología Neotropical (III COPANEO); The Biologist (Lima), vol 10 (2), Suplemento Especial 2; 2012 Noviembre 5 al 10; Lima, Perú; 2012.

15. Montalvo-Sabino, E, Cipriano-Fonseca, F, Marcelo-Andrade, E, RosasJara, DM, Mines-Huaman, WM, Capcha-Tucto, LN, Chávez-Chávez, C, Benites-Mendoza, B, Sandoval-Tolentino, M, Pineda-Castillo, CA, Cárdenas - Callirgos, J, Wetzel, EJ \& lannacone, J. Factors associated with contamination of public parks (Huanuco, Peru) by Toxocara canis eggs and other endoparasites of zoonotic importance. Neotrop. Helminthol. 2014;8(2):259-268.

16. Cárdenas-Callirgos, J., C. Mendoza, \& E. Wetzel. 2012. Intestinal parasites in indigenous communities of Santa Cruz and Chirick Sacha near Tarapoto (El Dorado region), San Martin. En: lannacone, J. \& CárdenasCallirgos, J. (Eds). 2012. III Congreso Internacional de Parasitología Neotropical (III COPANEO); The Biologist (Lima), vol 10 (2), Suplemento Especial 2; 2012 Noviembre 5 al 10; Lima, Perú; 2012.

17. Cárdenas - Callirgos, Jorge Manuel; Espinoza - Villanueva, Vicente; van Oordt - La Hoz, Francis; Sáenz - Montoya, Patrícia; Pineda - Castillo, Carlos; Rojas - Bravo, Violeta; Mendoza - Vidaurre, Carlos; Chávez - Cabello, Rocío; Tafur - Zevallos, Lisandro; Fonseca - Livias, Abner; Vecco - Giove, Daniel; Reátegui - Bardales, Carmen; Huaccho - Rojas Juan; Del Águila - Pérez, Carlos Alberto; Iannacone - Oliver, José Alberto \& Wetzel, Eric J. Impacto del Desarrollo Sostenible en la Salud Global Publica y Ambiental en el Perú: Experiencias de Campo. En: I ICGEH - I International Congress on Global Environmental Health; 2015 Marzo 12 al 14; Lima, Peru; 2015.

Consulte la Versión Electrónica de la Revista: Facultad de Medicina Humana Universidad Ricardo Palma

http://revistas.urp.edu.pe/index.php/RFMH<smiles>C1C2CC3CC1C3C2</smiles>

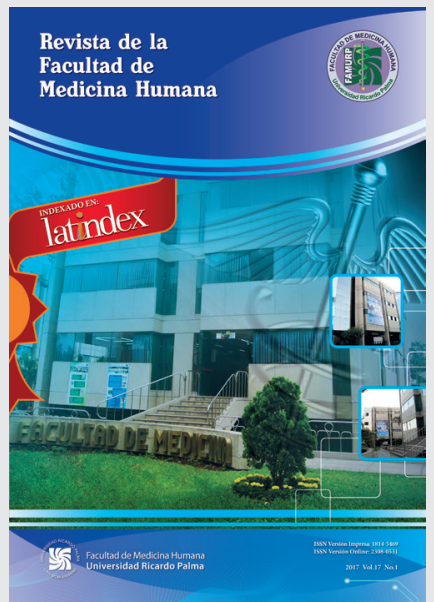

